\title{
Optimization of the Lipase-Catalyzed Selective Amidation of Phenylglycinol
}

\author{
Meina Sun ${ }^{1,2}$, Kaili Nie ${ }^{1,2 *}$, Fang Wang ${ }^{1,3}$ and Li Deng ${ }^{1,2 *}$ \\ ${ }^{1}$ Beijing Bioprocess Key Laboratory, Beijing University of Chemical Technology, Beijing, China, ${ }^{2}$ Amoy-BUCT Industrial \\ Bio-technovation Institute, Xiamen, China, ${ }^{3}$ State Key Laboratory of Chemical Resource Engineering, Beijing University of \\ Chemical Technology, Beijing, China
}

OPEN ACCESS

Edited by:

Zhi-Qiang Liu,

Zhejiang University of

Technology, China

Reviewed by:

Jiufu Qin,

Technical University of

Denmark, Denmark

Zhongyu You,

Jiaxing University, China

Yucai He,

Changzhou University, China

Xue Cai,

Zhejiang University of

Technology, China

*Correspondence:

Kaili Nie

niek@@mail.buct.edu.cn

Li Deng

dengli@mail.buct.edu.cn

Specialty section

This article was submitted to

Industrial Biotechnology,

a section of the journal

Frontiers in Bioengineering and

Biotechnology

Received: 15 August 2019 Accepted: 30 December 2019

Published: 22 January 2020

Citation:

Sun $M$, Nie K, Wang F and Deng L

(2020) Optimization of the

Lipase-Catalyzed Selective Amidation

of Phenylglycinol.

Front. Bioeng. Biotechnol. 7:486.

doi: 10.3389/fbioe.2019.00486
Ceramides and their analogs have a regulatory effect on inflammatory cytokines expression. It was found that a kind of ceramides analog synthesized from phenylglycinol could inhibit the production of cytokine TNF- $\alpha$. However, two active hydrogen groups are present in the phenylglycinol molecule. It is difficult to control the process without hydroxyl group protection to dominantly produce amide in the traditional chemical synthesis. A selective catalytic the amidation route of phenylglycinol by lipases was investigated in this research. The results indicated that the commercial immobilized lipase Novozym 435 has the best regio-selectivity on the amide group. Based on the experimental results and in silico simulation, it was found that the mechanism of specific $\mathrm{N}$-acyl selectivity of lipase was not only from intramolecular migration and proton shuttle mechanism, but also from the special structure of active site of enzyme. The optimal reaction yield of aromatic amide compound in a solvent-free system with lipase loading of $15 \mathrm{wt} \%$ (to the weight of total substrate) reached $89.41 \pm 2.8 \%$ with very few of byproducts detected $(0.21$ $\pm 0.1 \%$ ester and $0.64 \pm 0.2 \%$ diacetylated compound). Compare to other reported works, this work have the advantages such as low enzyme loading, solvent free, and high $\mathrm{N}$-acylation selectivity. Meanwhile, this Novozym 435 lipase based synthesis method has an excellent regio-selectivity on most kinds of amino alcohol compounds. Compared to the chemical method, the enzymatic synthesis exhibited high regio-selectivity, and conversion rates. The method could be a promising alternative strategy for the synthesis of aromatic alkanolamides.

Keywords: lipase, ceramides analogs, solvent-free, phenylglycinol, amidation

\section{HIGHTLIGHTS}

- Enzymatic amidation of phenylglycinol and capric acid in solvent-free system.

- Novozyme 435 has effective regio-selectivity for the amidation of phenylglycinol.

- The special structure of enzyme active site influenced regio-selectivity of reaction.

- Enzymatic method is an alternative synthetic strategy for aromatic alkanolamides.

\section{INTRODUCTION}

Ceramides are a family of waxy lipid molecules. Normally, they contain a sphingosine and a long chain fatty acid (Long et al., 2006; Sugiyama et al., 2010). Ceramides are found in high concentrations within the cell membrane of eukaryotic cells and within some kinds of plant tissues (Morad and Cabot, 2013). Natural ceramides have a variety of physiological functions, such as 


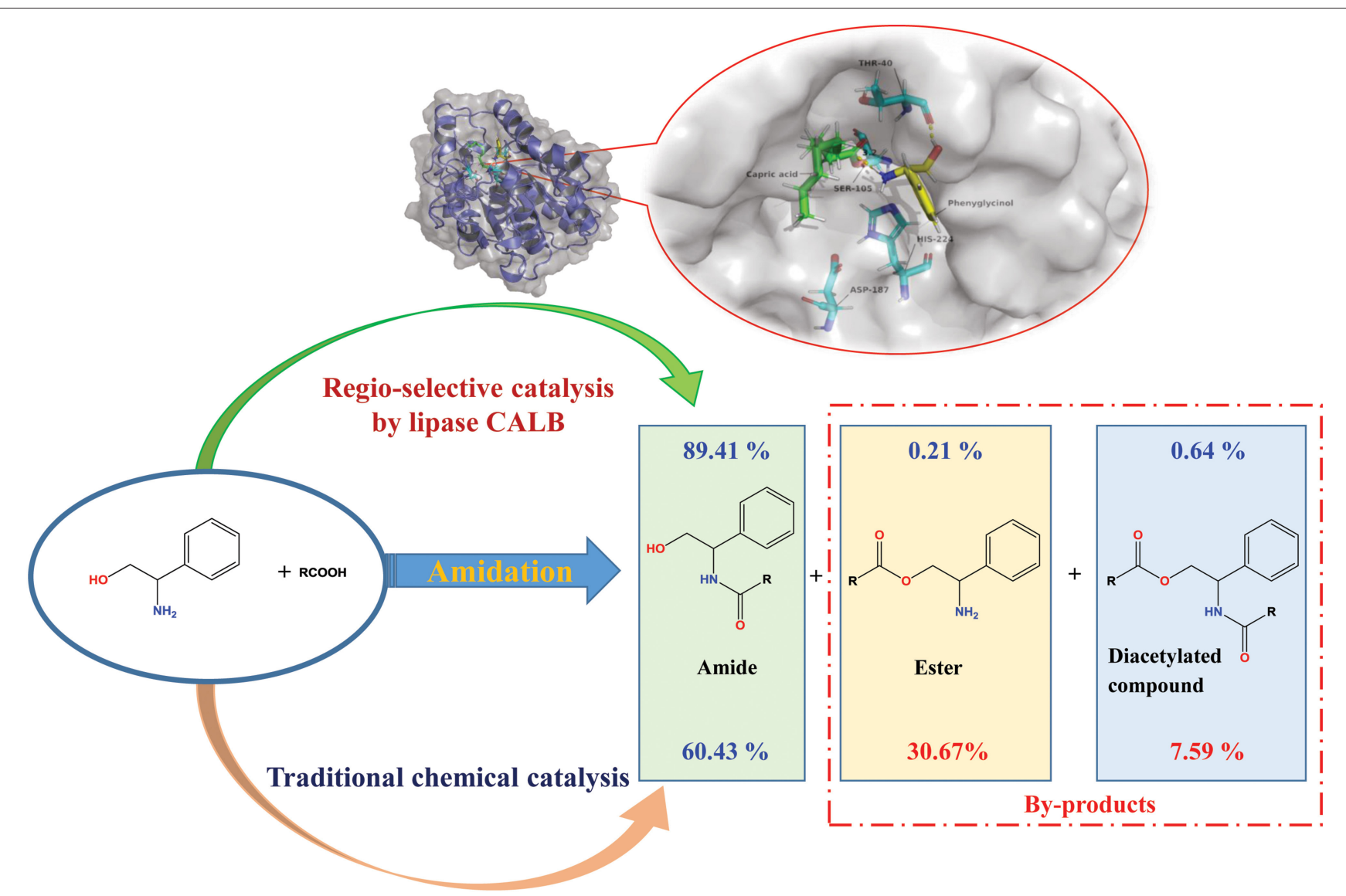

Graphical Abstract | Lipase-catalyzed selective amidation of phenylglycinol.

their anti-tumor and signal transduction activities, their regulation of apoptosis, their immunity promotion, and their anti-inflammatory properties (Müller et al., 2004). The natural ceramide structure is unstable and difficult to be synthesized.

Over the years, it was found that a series of structuresimilar molecules have the physiological activity of ceramides. Parts of those ceramide (Jiang et al., 2016; He and Schuchman, 2018) analogs could act as the inhibiter of cytokines, thus have the potential medicinal properties. Matsui et al. and Toshiaki et al. reported a class of small ceramide analog molecules that could inhibit the production of cytokine TNF- $\alpha$ (Matsui et al., 2002; Toshiaki et al., 2002) (Scheme 1). One of the synthetic routes of compound $\mathbf{1}$ was used with phenylglycinol as substrate. However, the phenylglycinol molecule contains two active hydrogen groups. It is difficult to control the process without hydroxyl group protection to dominantly produce amide (Andrea et al., 2019). By-products, such as esters and diacetylated compound, would reduce the yield of the amide product.

The traditional chemical catalysis has poor regio-selectivity for the reaction of Scheme 1. It will form a certain amount of by-products. Compared to chemical catalysts, enzymes are more effective toward the substrate specificity and regio-selectivity (Bornscheuer, 2005). Among the enzymes, lipase (EC 3.1.1.3) was often applied for the esterification and amidation reactions
(Kirchner et al., 1985; Martinek and Semenov, 2002; Vicente, 2002; Slotema et al., 2003; Tan, 2010). Due to the excellent regio-selectivity, high efficiency and environment amity, many works about lipase, especially commercial lipase Novozym 435, synthesizing high value-added amide products, applied for drug intermediates and cosmetic materials have been reported (Levinson et al., 2005; Martínková et al., 2008; Baum et al., 2011; Schwab et al., 2013; Stavila et al., 2013; Liñares et al., 2014). Among all the researches, amino alcohols have two active hydrogen groups, the amidation reaction on which have been well studied (Syrén et al., 2012; Wang et al., 2015). Based on the results of those researches, it was found that the reactions have outstanding regio-selectivity of $\mathrm{N}$-acylation products. Such as Fernandez-Perez et al. studied the enzymatic synthesis of amide surfactants from ethanolamine, with a good regio-selectivity and yield of $92 \% \mathrm{~N}$-acyl ethanolamine demonstrated in $1.5 \mathrm{~h}$ under the optimal conditions (Fernandez-Perez and Otero, 2001; Irimescu and Kato, 2004). The reason of the regioselectivity of lipase might be due to two aspects. Firstly, it has been suggested that the lipase-catalyzed $\mathrm{N}$-acylation of amino alcohols proceeded through initial O-acylation followed by spontaneous $\mathrm{O}$ - to $\mathrm{N}$-acyl migration (Tufvesson et al., 2006). Secondly, Syrén et al. proposed a proton shuttle reaction mechanism, in which two protons are transferred in the transition state coincidently with the nucleophilic attack, and it 


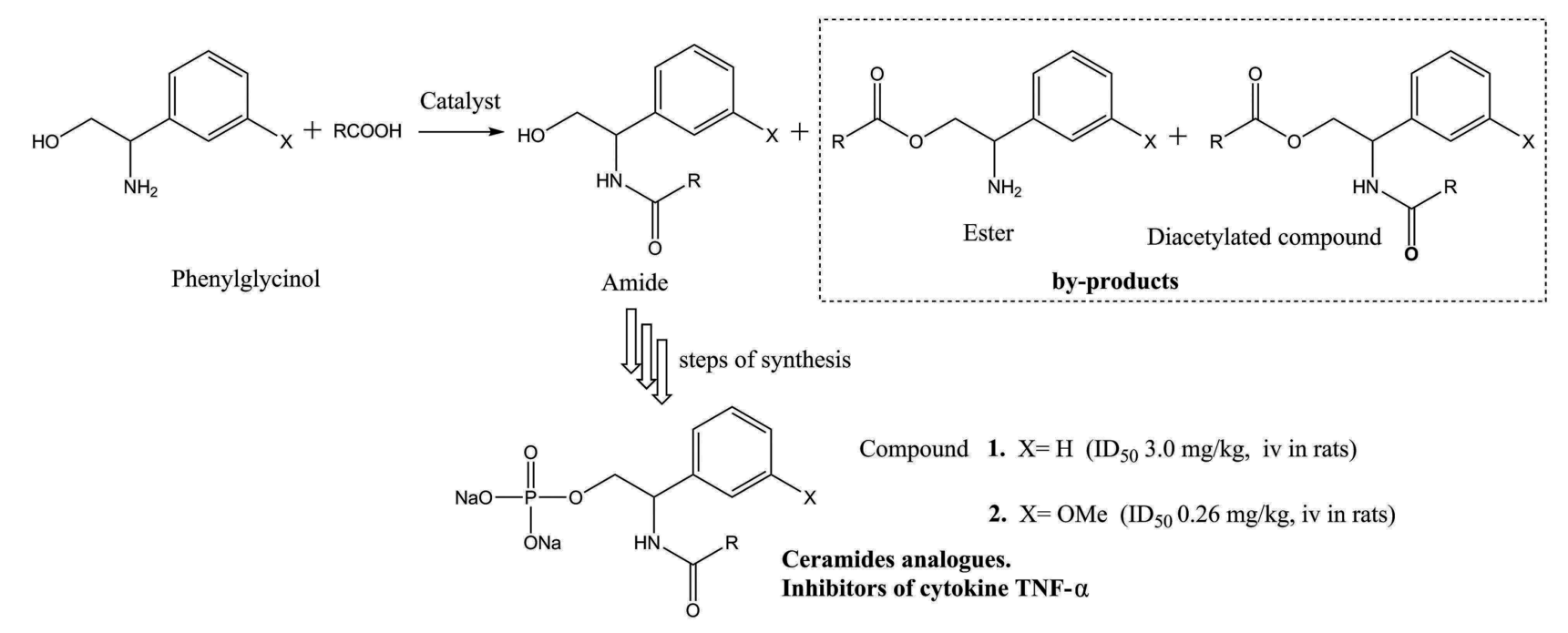

SCHEME 1 | Ceramides analogs synthesize from phenylglycinol.

would enhance reaction rate for enzyme-catalyzed $\mathrm{N}$-acylation (Syrén et al., 2012).

Even lots of researches have carried out the investigation of lipase catalyzed the $\mathrm{N}$-acylation of amino alcohols, there were few reports about the lipase catalyzed the acylation of phenylglycinol and its similar structure molecules. In this paper, we aimed to utilize lipase to control the selectivity of the amidation of phenylglycinol. The process was optimized and the mechanism of lipase selectivity was explored with the molecular simulation in silico. Furthermore, the feasibility of the method for the substrates with the similar structure to phenylglycinol was also investigated.

\section{MATERIALS AND METHODS}

\section{Materials}

Phenylglycinol and capric acid were obtained from Macleans, Shanghai, China. Bio-catalysts of Novozym435 lipase, Lipozyme RM IM, and Lipozyme TL IM were obtained from Novozymes, Beijing, China. Candida sp. 99-125 was purchased from Beijing CTA New Century Biotechnology Co. LTD., Beijing, China, and the lipase was immobilized on diatomaceous earth. The fermentation and immobilization procedure of the lipase were described in our previous research (Tan et al., 2003; Deng et al., 2005). All other chemicals used were of analytical grade, and used as obtained.

\section{Enzymatic Amidation Reactions}

Raw materials of phenylglycinol and capric acid were dissolved for homogeneous mixing at $600 \mathrm{rpm}, 78^{\circ} \mathrm{C}$, and the substrate molar ratio was set from 1:1 to 1:1.5. After temperature cooling to $60^{\circ} \mathrm{C}$, the bio-reaction was carried out for $19 \mathrm{~h}$ along with the lipase addition. Moreover, the dosage of the lipase was ranged from 0 to $25 \mathrm{wt} \%$ in this reaction. The target production was purified through thin layer chromatography plates and column chromatography that was analyzed by gas chromatography.

\section{Gas Chromatography}

Samples were analyzed by gas chromatography (GC, Shimadzu GC-2010 plus, Japan), which was equipped with FID flame ionization detector. The column was DB- 1 capillary column $(30 \mathrm{~m} \times 0.25 \mathrm{~mm} \times 0.1 \mu \mathrm{m}$, Agilent). Nitrogen with a flow rate of $1.22 \mathrm{~mL} / \mathrm{min}$ was used as carrier gas for the GC analysis. The column was set at $100^{\circ} \mathrm{C}$ for $0.2 \mathrm{~min}$, first increased to $220^{\circ} \mathrm{C}$ at a rate of $8^{\circ} \mathrm{C} / \mathrm{min}$, then increased to $310^{\circ} \mathrm{C}$ at a rate of $12^{\circ} \mathrm{C} / \mathrm{min}$ and maintained for $3 \mathrm{~min}$, finally increased to $370^{\circ} \mathrm{C}$ at a rate of $20^{\circ} \mathrm{C} / \mathrm{min}$ and maintained for $5 \mathrm{~min}$. The injector and the detector temperature were set at $370^{\circ} \mathrm{C}$. Each assay was validated at least triplicated for mean values, with margin of error lower than $5 \%$.

\section{Identification of the Products by FT-IR, ${ }^{1} \mathrm{H}$ NMR, and GC-MS}

Each component was isolated by crystallization in methanol at $-20^{\circ} \mathrm{C}$, and the product identification was operated by ${ }^{1} \mathrm{H} \mathrm{NMR}$, FT-IR and GC-MS.

\section{${ }^{1}$ H NMR}

Structure of the purified product was analyzed by ${ }^{1} \mathrm{H}$ NMR spectra (NMR, Avance III $400 \mathrm{MHz}$, Switzerland) with solvent of $\mathrm{CDCl}_{3}$. The obtained spectra had verified the product structure with the signals of $87.28-7.38\left(\mathrm{~m}, 5 \mathrm{H}, \mathrm{C}_{6} \mathrm{H}_{5}\right), 6.08(\mathrm{br} \mathrm{s}, 1 \mathrm{H}, \mathrm{NH})$, 5.05-5.10 ( $\mathrm{m}, 1 \mathrm{H}, \mathrm{C}_{6} \mathrm{H}_{5} \mathrm{CH}$ ), 3.93 (ddd, $2 \mathrm{H}, \mathrm{CH}_{2} \mathrm{OH}$ ), 2.61 (br s, $1 \mathrm{H}, \mathrm{OH}), 2.25\left(\mathrm{t}, 2 \mathrm{H}, \mathrm{CO} \mathrm{CH}_{2}\right), 1.60-1.70(\mathrm{~m}, 2 \mathrm{H}), 1.20-1.35(\mathrm{~m}$, $\left.12 \mathrm{H}, \mathrm{C}_{2-7}\right), 0.88\left(\mathrm{~m}, 3 \mathrm{H}, \mathrm{C}_{8} \mathrm{CH}_{3}\right)$, whose figure had been showed in Figure S1. 
TABLE 1 | Effect of different lipases on the amidation reaction.

\begin{tabular}{lcccc}
\hline Enzymes & $\begin{array}{c}\text { Phenylglycinol } \\
\text { consumption/ } \\
\text { wt\% }\end{array}$ & $\begin{array}{c}\text { Amide/ } \\
\text { wt\% }\end{array}$ & $\begin{array}{c}\text { Ester/ } \\
\text { wt } \%\end{array}$ & $\begin{array}{c}\text { Diacetylated } \\
\text { compound/ } \\
\text { wt } \%\end{array}$ \\
\hline Novozym 435 & $41.24 \pm 4.32$ & $37.89 \pm 3.03$ & $1.42 \pm 0.51$ & $1.95 \pm 0.64$ \\
Candida sp. 99-125 & $27.94 \pm 3.20$ & $18.87 \pm 4.87$ & $6.16 \pm 3.68$ & $3.91 \pm 2.10$ \\
Lipozyme TL IM & $27.11 \pm 2.31$ & $18.56 \pm 2.68$ & $7.65 \pm 2.61$ & $3.43 \pm 1.44$ \\
Lipozyme RM IM & $32.68 \pm 4.16$ & $13.35 \pm 1.85$ & $16.22 \pm 2.42$ & $2.10 \pm 1.35$
\end{tabular}

Reaction conditions: phenylglycinol and capric acid were added at certain molar ratio, the enzyme loading was $10 \mathrm{wt} \%$. The reaction was incubated at $55^{\circ} \mathrm{C}$ for $10 \mathrm{~h}$ with tert-amyl-alcohol as solvent

\section{FT-IR}

Functionalization of the purified product was analyzed by Fourier transform infrared spectroscopy (FT-IR, Model Nicolet 5ZDX, America) in the range from 400 to $4,000 \mathrm{~cm}^{-1}$. The detection results were listed in Figure S2.

\section{Gas Chromatography Mass Spectrometry}

Gas chromatography mass spectra (GC-MS Agilent GC-MS 7890A-5975C) was chosen as the instrument for the product identification. GC-MS analysis was carried out with the column of HP-5 MS capillary column $(30 \mathrm{~m} \times 0.25 \mathrm{~mm} \times 0.1 \mu \mathrm{m}$, Agilent). Helium was used as carrier gas, with a flow rate of 1.22 $\mathrm{mL} / \mathrm{min}$. The column temperature was set at $100^{\circ} \mathrm{C}$ for $1 \mathrm{~min}$, then increased to $300^{\circ} \mathrm{C}$ at a rate of $10^{\circ} \mathrm{C} / \mathrm{min}$, and maintained for $15 \mathrm{~min}$. Mass spectrum was set with ionization voltage of $70 \mathrm{eV}$, and ion source temperature of $200^{\circ} \mathrm{C}$. Assay result was reflected in Figures S3-S23.

\section{Assay of in silico Simulation}

The initial opened conformation of YLLIP2, based on the X-ray structure of YLLIP2 (PDB ID: 4JEI), was obtained in our previous study (Cao et al., 2014). Other enzymes were used with their X-ray structure download from PDB protein date bank (http://www.rcsb.org/, Candida. antarctic lipase, PDB code: 5GV5; Thermomyces lanuginosus lipase, PDB code: 1EIN; Rhizomucor miehei lipase, PDB code: 4TGL, respectively). All docking simulations were performed with the YASARA software package (version 16.3.8). AMBER03 force field were used, and docking simulation were carried out at $313 \mathrm{~K}$ and atmospheric pressure. Standard docking_run macro (http://www.yasara. org/macros.html) of YASARA was used for simulation. The docking results were viewed and analyzed with PyMOL package (https://pymol.org/).

\section{RESULTS AND DISCUSSION}

\section{Effect of Enzymes on the Amidation Reaction}

The modification of only amino group in the substrate molecule is a fundamental challenge of this work. Due to In this work, the conversion rates and regio-selectivity of four kinds of immobilized lipases were investigated.

The results in Table 1 indicated that compared to other lipases, lipase Novozym 435 (Candida antarctic lipase B, CALB) exhibits the best reactivity and regio-selectivity. Normally, when there are more than one functionalities in a substrate which is susceptible to acetylation, the unwanted groups should be protected. However, the results of CALB catalysis (Table 1) indicated that the aim product could be synthesized without group protection. In the early researches about acylation of ethanolamine, Skorey et al. and Tufvesson et al. considered the $\mathrm{N}$-acylation selectivity was through an intramolecular Oto $\mathrm{N}$-acyl migration (Skorey et al., 1987; Tufvesson et al., 2006). However, the migration was based on the balance of thermodynamics (Fodor and Kiss, 1950). It is difficult to explain the reason that different lipases have the various ratios of amide to ester product. Syrén et al. explained the phenomenon as the proton shuttle mechanism (Syrén et al., 2012). Nevertheless, it seemed that the reason was not fit for the results of Lipozyme RM IM (Table 1, line 4). To better understand the mechanism of the different regio-selectivity of various lipases as catalyst in the reaction, a molecular docking process in silico was performed. The simulation result of CALB (Figure 1A) indicated that the hydrophobic interaction between the benzene ring and residues of Ile-258, Ala-282, Leu-278, and Ile-189 would maintain the conformation of phenylglycinol. Meanwhile, the hydrogen bonding between the hydroxyl group and Thr-40 makes the amino group close to the active site (Ser-105), and could attack the carbonyl group of the lipase-acylation intermediate (the distance is $3.2 \AA$ ). However, the simulation results of Candida sp. (Yarrowia lipolytica lipase, Figure 1B), and Lipozyme TL IM (Thermomyces lanuginosus lipase, Figure 1C) indicated that the hydroxyl and amino group of phenylglycinol have the similar distance to the active site. Whereas, the hydroxyl group of phenylglycinol was much closer to the active site than the amino group in the result of Lipozyme RM IM (Rhizomucor miehei lipase, Figure 1D). Thus, those three lipases lacked a regio-selectivity in the reaction. In addition, CALB has the best simulation result of binding energy $(-6.01 \mathrm{kcal} / \mathrm{mol})$, which indicated that the active site of CALB lipase is more fit for combination with phenylglycinol than other lipases. Normally, different lipases have specific regio-selectivity due to the special structure of substrate binding domains (Wang et al., 2015). The dominant literatures about lipases catalyzed the acylation of ethanolamine used Novozym 435 (CALB) as catalyst. The protein sequence alignment indicates that the homology of CALB is quite different from other three lipases (Figure S24), and the results of protein structure alignment also verified the structure difference of CALB from others (Figure S25). Based on the results of our experiments and in silico docking simulation, it was considered that the mechanism of specific $\mathrm{N}$-acyl selectivity of lipase was not only from intramolecular migration and proton shuttle mechanism, but might be mainly from the special structure of enzyme active site. Therefore, Novozym 435 was chosen as the catalyst for further investigation.

\section{Effect of Enzyme Amount on the Amidation Reaction}

The enzyme loading is one of the key factors in the enzymatic reactions. A series of enzyme loadings was carried out and the 

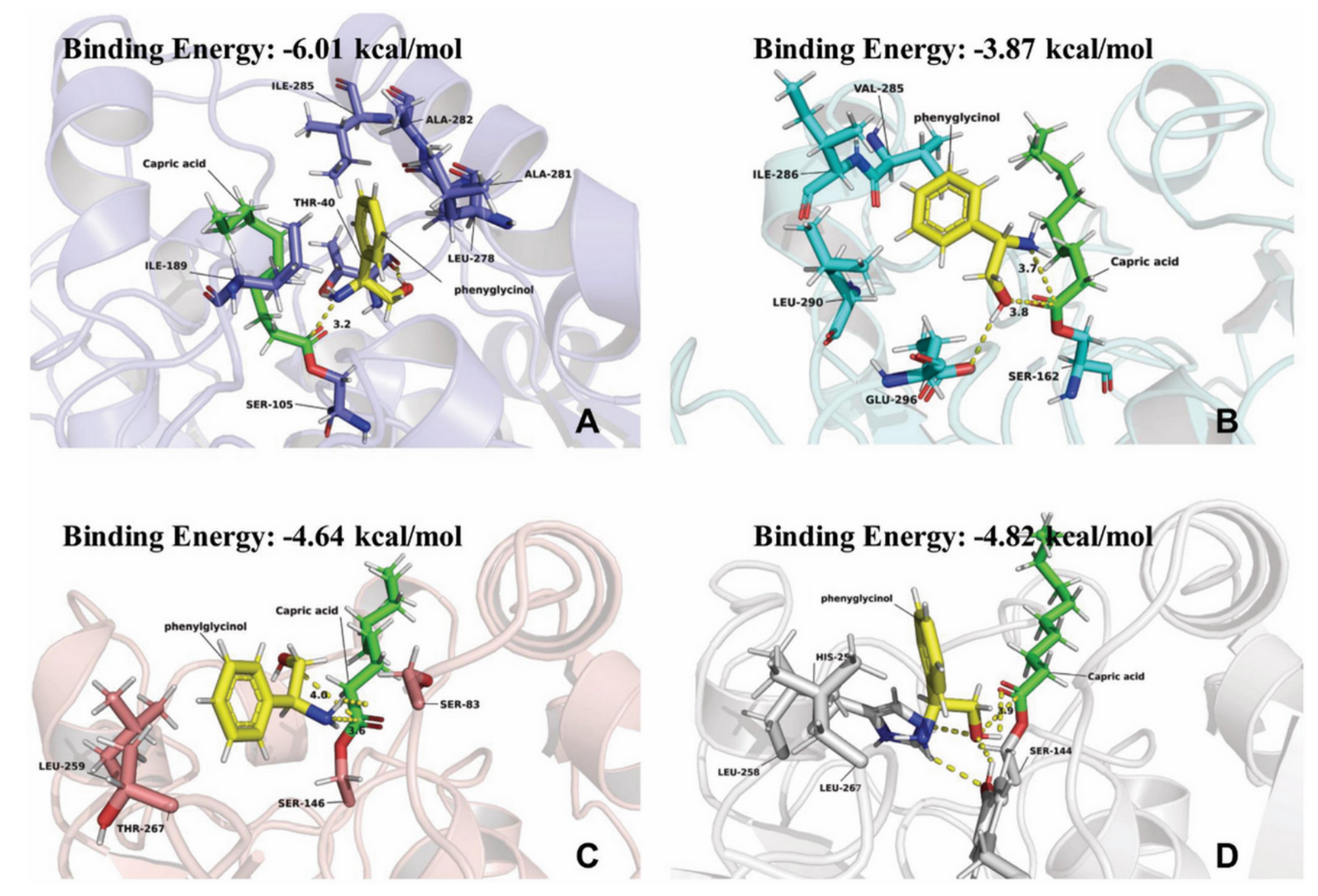

FIGURE 1 | Simulation results of phenylglycinol docking into active site of lipases. (A) Candida. antarctic lipase B (colored in slate, PDB code: 5GV5). (B) Yarrowia lipolytica lipase 2 (colored in cyan, PDB code: 4JEI). (C) Thermomyces lanuginosus lipase (colored in salmon, PDB code: 1EIN). (D) Rhizomucor miehei lipase (colored in light gray, PDB code: 4TGL). Phenylglycinol was colored in yellow. Capric acid was colored in light green.

results were illustrated in Figure 2. Without the addition of enzyme, the amidation reaction of phenylglycinol with capric acid could not be initiated. In the enzymatic groups, the enzyme loadings exhibited no significant differences at the initial $8 \mathrm{~h}$. As the reaction time prolonged, the higher loadings of enzyme facilitated the reaction. When the enzyme loading exceeded 15 $w t \%$, the yield remained stable. This is because of the effective binding of substrates and enzyme active sites would accelerate the reactions. Less or excessive enzyme loadings meant that the availability of active sites of enzymes was inadequate or not properly exposed to the substrates. In addition, excessive enzyme loadings could increase the viscosity of the reaction system, which might prevent efficient mass transfer and result in low catalytic efficiency. Therefore, enzyme loading of $15 \mathrm{wt} \%$ is the optimal and economic choice.

\section{Effect of Solvents and Temperature on the Amidation Reaction}

The role of organic solvents in the enzymatic reaction is to increase the solubility of the substrates and to ensure sufficient contact between the enzyme active sites and the substrates
(Fernandez-Perez and Otero, 2003; Levinson et al., 2005; Xiao et al., 2013). However, the organic solvents and temperature also showed great influence on the enzyme activity, stability and selectivity (Zaks and Klibanov, 1984; Ragupathy et al., 2012; Garcia-Alles and Gotor, 2015). The solvent-free system is a greener and safer alternative (Prasad et al., 2005; Ali et al., 2013), but would increase the viscosity of the system. The comparison and selection of solvents was carried out and shown in Figure 3.

A series of organic solvents with different $\log P$ were screened for the lipase-catalyzed the amidation reactions. $\log P$ of benzene, tert-butanol, tert-amyl alcohol, dichloromethane, and acetonitrile were $2.0,0.6,1.3,1.01$, and 0.33 , respectively. The lower the $\log P$-values, the more hydrophobic the solvents would be. The $\log P$ of benzene is 2.0 , which is beneficial to maintain the activity of the enzyme, but the hydrophobicity is unfavorable to the substrate dissolution. The $\log P$ of tert-amyl alcohol is 1.3 , which is favorable for dissolution of the substrates, but it competes with the essential free water of the enzyme molecule to maintain the catalytic activity, thereby reducing the enzyme activity (Lee and Parkin, 2001; Gorman and Dordick, 2010). Even though the enzymatic synthesis using free fatty acid as acyl donor 


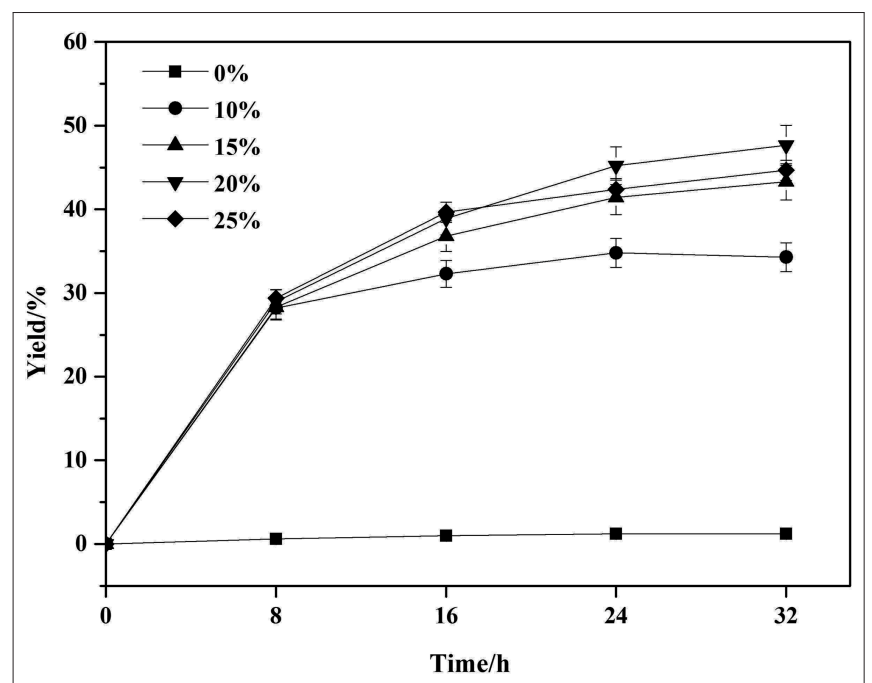

FIGURE 2 | Effect of Novozym 435 amounts on the amidation reaction. Enzymatic reaction conditions: molar ratio of phenylglycinol to capric acid at $1: 1$, and Novozym 435 loadings varied from 0 to $25 \mathrm{wt} \%$. The reaction was carried out at $40^{\circ} \mathrm{C}$ for $32 \mathrm{~h}$, with tert-amyl-alcohol as solvent.

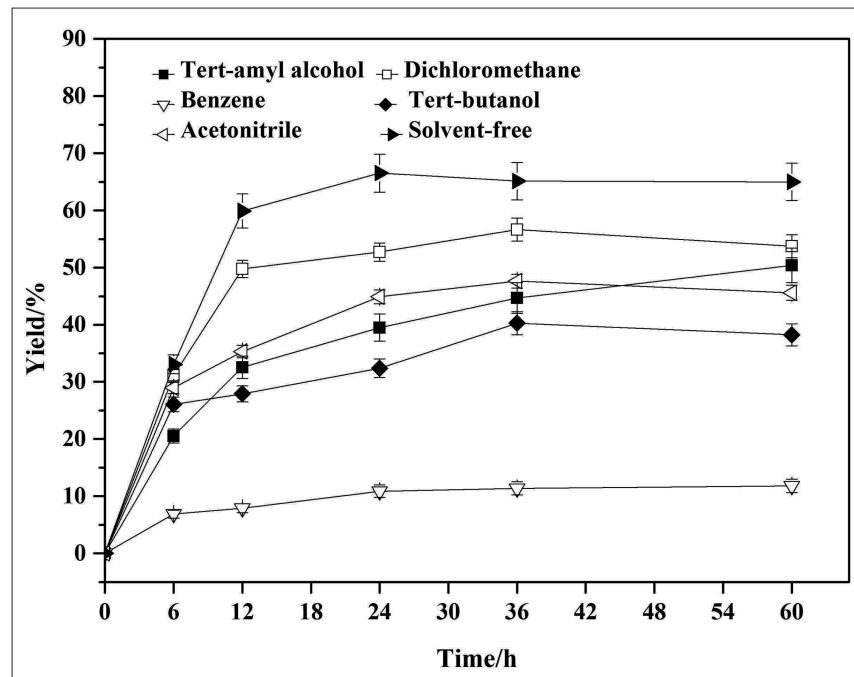

FIGURE 3 | Effect of solvents on the amidation reaction. Enzymatic reaction conditions: molar ratio of phenylglycinol to capric acid at 1:1, and Novozym 435 loading was $15 \mathrm{wt} \%$. The reaction was carried out at $40^{\circ} \mathrm{C}$ for $60 \mathrm{~h}$.

is a relatively effective process, the amidation reaction conducted in a solvent usually needs $30 \%$ (w/w, relative to total reactants) lipase or more (Plastina et al., 2009; Wang et al., 2012, 2013). The high lipase load maybe attributed to the formation of undissolved ion pair from equivalent moles of amine and free fatty acid, and the lipase has a low catalytic activity to ion pair. In the solvent-free system (Prasad et al., 2005), capric acid with the weak polarity could dissolve phenylglycinol completely. This would increase the contact area of the raw material and the enzyme, which would promote the reaction. Therefore we investigated the feasibility of using solvent-free system. The solvent-free system

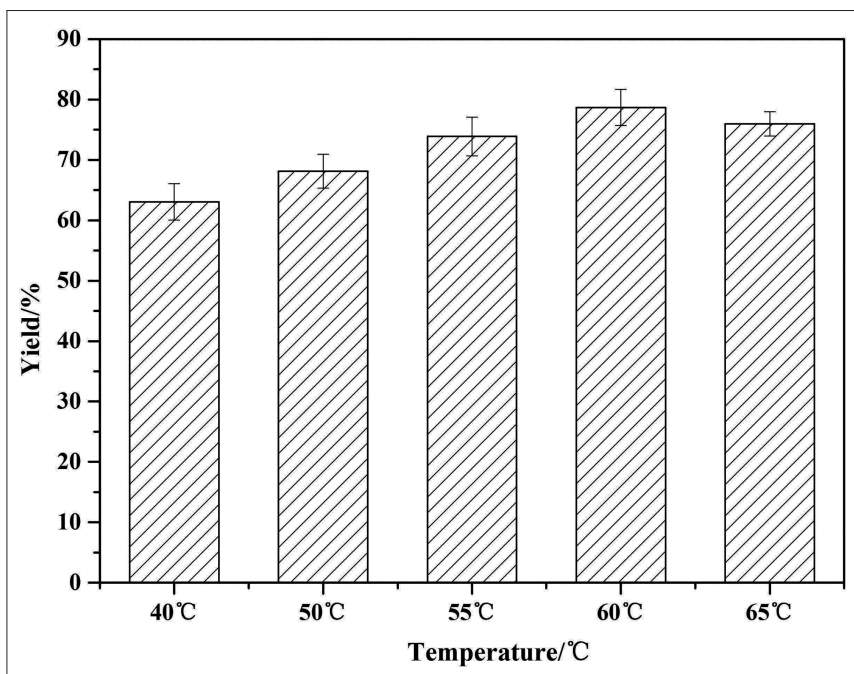

FIGURE 4 | Effect of temperature on the amidation reaction. Enzymatic reaction conditions: a molar ratio of phenylglycinol to capric acid at 1:1, and Novozym 435 loading of 15 wt\%. Solvent-free systems for $24 \mathrm{~h}$ in a varied but constant temperature mixer.

was superior to the applied solvents. Taking the process safety and post-process separation into consideration, the solvent-free system is a better choice. The lipase demonstrates good catalytic efficiency in a solvent-free system. Meanwhile, further investigate about catalyst dosage indicated that $15 \mathrm{wt} \%$ was the best of enzyme loading for the solvent-free system (Figure S26).

The optimization of temperature was presented in Figure 4. The lipase activity increases with the increase of reaction temperature in the beginning. The higher temperature can decrease the viscosity of the system, promoting the mass transfer efficiency. The yield increased from 65 to $81 \%$ when the temperature rose from 40 to $60^{\circ} \mathrm{C}$. As the temperature continued to rise to $65^{\circ} \mathrm{C}$, the yield began to decrease. Higher temperature than $60^{\circ} \mathrm{C}$ led to the denaturation of the enzyme, resulting in a decreased product yield. Therefore, the optimal temperature for solvent-free reaction is $60^{\circ} \mathrm{C}$.

\section{Effect of Substrates Molar Ratio on the Amidation Reaction}

Different molar ratios of phenylglycinol to capric acid were investigated $(1: 2,1: 1,1.2: 1,1.5: 1,1.7: 1)$ and the results were shown in Figure 5. The ratio of reactants was crucial for selectivity of the enzyme (Maugard et al., 1997). Excess capric acid caused the amino moiety in phenylglycinol to be protonated. Therefore, the amide reaction may be inhibited, resulting in the formation of by-product diacetylated compound. When the molar ratio of phenylglycinol to capric acid increased, the yield of phenylglycolamide increased sharply and by-product diacetylated compound decreased. Excessive phenylglycinol could slow down the formation of ion pairs and inhibit $\mathrm{N}$-acylation (Tufvesson et al., 2006). The $\mathrm{N}$ atom of phenylglycinol is less electronegative, while the amino group is more likely to provide electrons. Thus, the amino group has 


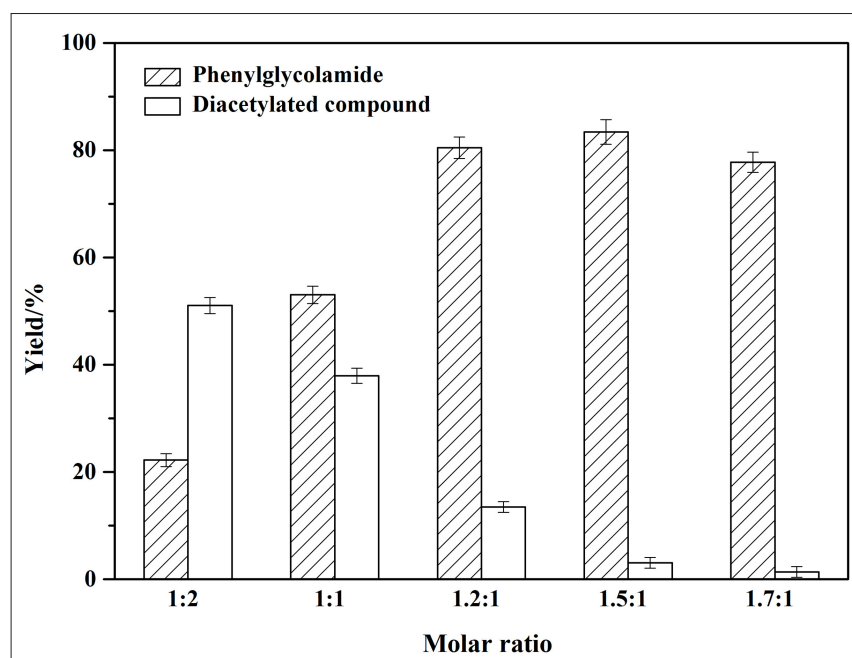

FIGURE 5 | Effect of substrates molar ratio on the amidation reaction. Enzymatic reaction conditions: different molar ratio of phenylglycinol to capric acid; Novozym 435 loading of 15 wt\%. Solvent-free systems were carried out at $60^{\circ} \mathrm{C}$ for $24 \mathrm{~h}$.

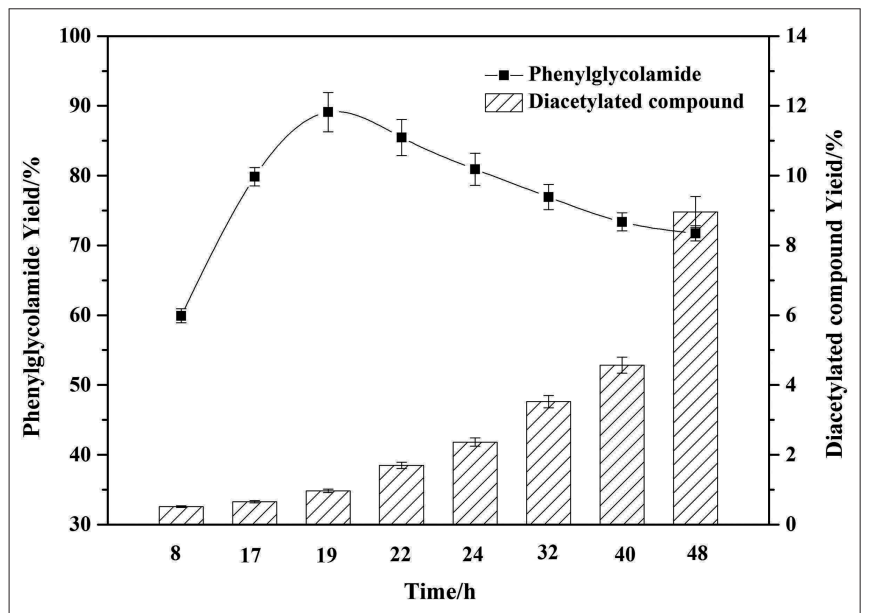

FIGURE 6 | Effect of reaction time on the amidation reaction. Enzymatic reaction conditions: molar ratio of phenylglycinol to capric acid of 1.5:1, and Novozym 435 loading of $15 \mathrm{wt} \%$. Solvent-free reactions at $60^{\circ} \mathrm{C}$ for different reaction times.

stronger nucleophilic ability than the hydroxyl group, and the activity of the amino hydrogen is stronger than that of hydroxyl group. In this work, it was found that the by-product yield could be controlled from regulation of the substrate molar ratio. The optimal molar ratio of phenylglycinol to capric acid is $1.5: 1$.

\section{Effect of Reaction Time on the Amidation Reaction}

As shown in Figure 6, different reaction times significantly affected the phenylglycolamide yield. The yield of ceramide reached $89.41 \%$ at $19 \mathrm{~h}$ and then began to decrease. Meanwhile, the by-product diacetylated compound began to accumulate. Reaction times in excess of $19 \mathrm{~h}$ might

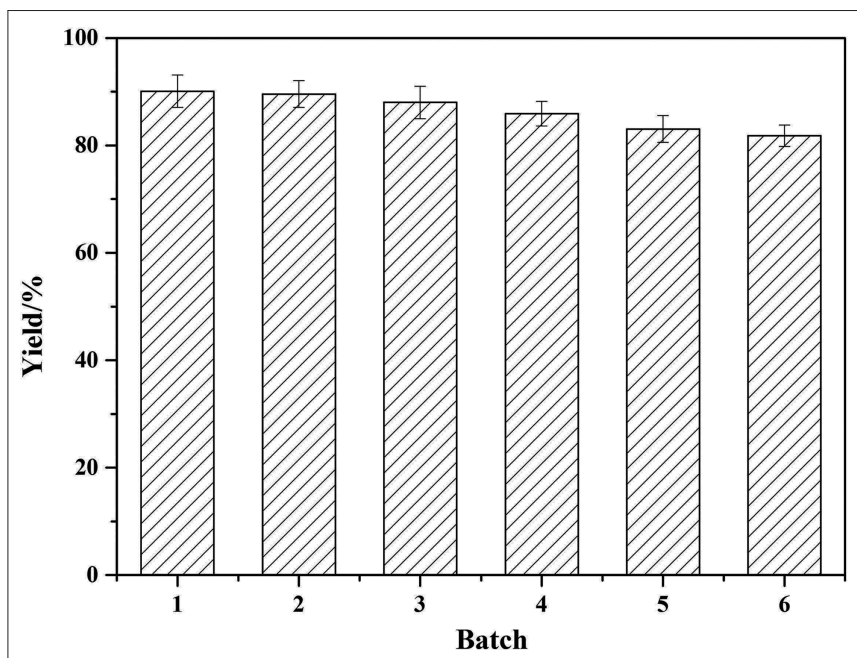

FIGURE 7 | Reusability of Novozym 435. Reaction conditions: molar ratio of phenylglycinol to capric acid of 1.5:1, Novozym 435 loading of 15 wt\%. Solvent-free reactions at $60^{\circ} \mathrm{C}$ for $19 \mathrm{~h}$. Subsequently, the enzyme was washed 3-5 times with hexane, blown dry, and collected for catalysis of the next experiment.

accelerate the consumption of the phenylglycolamide through polymerization or hydrolysis. Thus, $19 \mathrm{~h}$ reaction time is adequate for the reaction.

Compared with the previous chemical synthesis of phenylglycolamide, the chemical synthesis of phenylglycolamide required at least $24 \mathrm{~h}$ with a yield around 59\%. In the present work, the reaction time was reduced to $19 \mathrm{~h}$ and the yield was increased to $89.41 \%$. The enzymatic process was superior to the chemical process both in efficiency and environmental amity.

\section{Enzyme Reusability}

Enzyme reusability was investigated to evaluate the enzyme stability and the results were showed in Figure 7. As demonstrated, Novozym 435 has high catalytic efficiency and good stability under the reaction conditions. After one batch of reaction, the enzyme activity kept stable. Following six batches were repeated, and the yield still maintained about $82 \%$. This proved that the enzymatic process is of good potential for future industrial production.

\section{The Advantages and Generality of the Method}

Different kinds of amino alcohols (Scheme 2) were used as substrates to evaluate the generality of the regio-selectivity of lipase. The results (Table 2) indicated that for all kinds of substrates (a-h) tested in the experiment, Novozym 435 lipase exhibited specific regio-selectivity to the amino group, and an acceptable conversion. It could also be found that the property of substitute group (-R) would affect the regio-selectivity. Due to the strong electron-pulling effect, among all the substitute group, phenyl group exhibited the best impact. Meanwhile, it was found that CALB enzyme could catalyzed both R-, S-enantiomer of phenylglycinol with similar conversion rates (Table 2, results of 


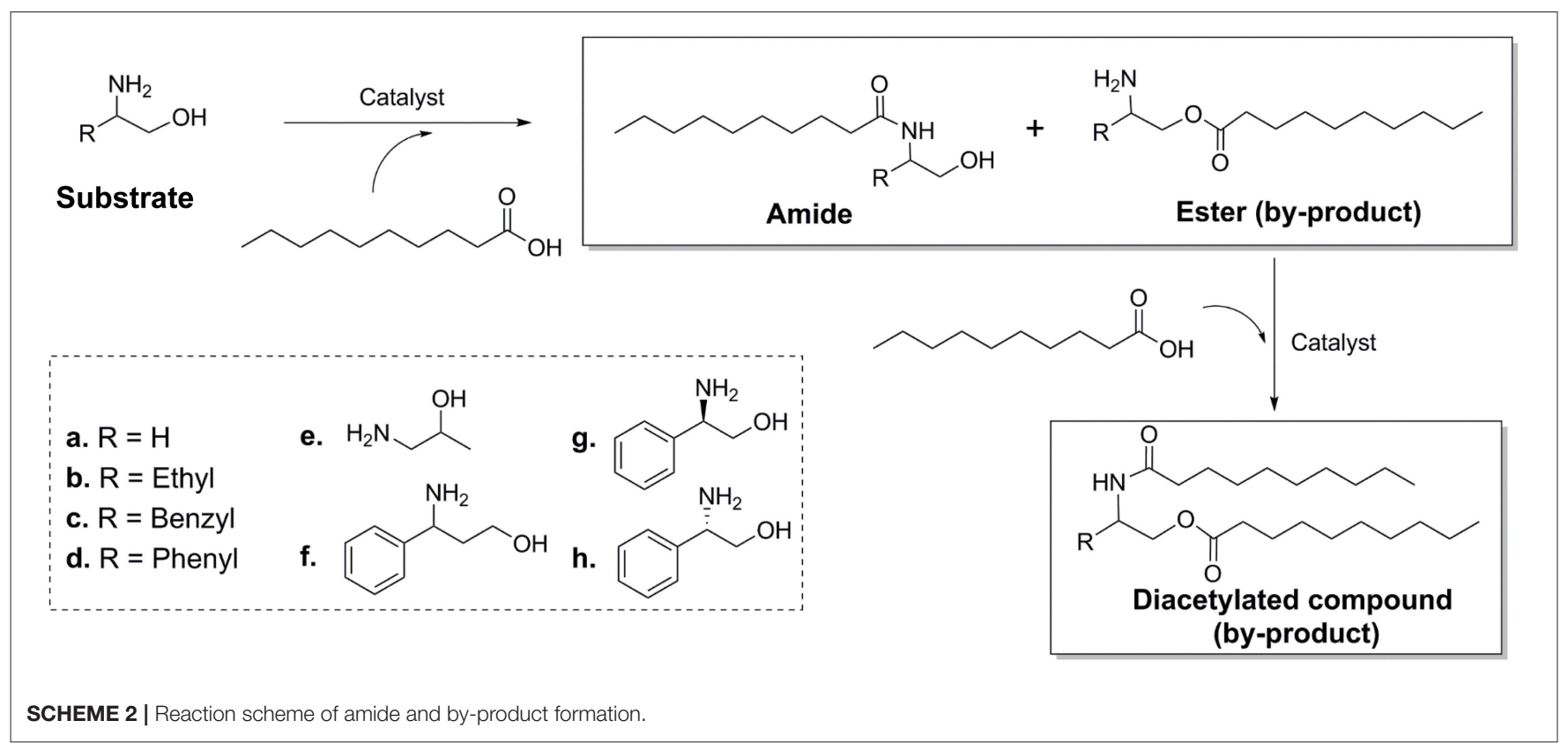

TABLE 2 | Effect of different methods on the amidation reaction with different kinds of substrate.

\begin{tabular}{lcccc}
\hline Methods & Substrates & $\begin{array}{c}\text { Amide/ } \\
\text { wt\% }\end{array}$ & $\begin{array}{c}\text { Ester/ } \\
\text { wt } \%\end{array}$ & $\begin{array}{c}\text { Diacetylated } \\
\text { compound/ } \\
\text { wt } \%\end{array}$ \\
\hline Enzyme catalysis & a & $79.68 \pm 2.9$ & $8.63 \pm 1.3$ & $9.77 \pm 1.0$ \\
& b & $63.68 \pm 1.5$ & $3.02 \pm 1.0$ & $5.43 \pm 0.7$ \\
& c & $67.73 \pm 1.6$ & $1.66 \pm 0.4$ & $3.24 \pm 0.6$ \\
& d $^{\text {a }}$ & $89.41 \pm 2.8$ & $0.21 \pm 0.1$ & $0.64 \pm 0.2$ \\
& e & $73.60 \pm 2.4$ & $4.73 \pm 1.2$ & $6.04 \pm 2.4$ \\
& f & $69.58 \pm 2.0$ & $0.56 \pm 0.1$ & $0.98 \pm 0.2$ \\
& g & $89.32 \pm 2.5$ & $0.18 \pm 0.09$ & $0.61 \pm 0.2$ \\
& $h^{\text {c }}$ & $89.35 \pm 2.9$ & $0.22 \pm 0.1$ & $0.65 \pm 0.1$ \\
\hline & a & $61.48 \pm 2.3$ & $27.23 \pm 1.9$ & $4.27 \pm 0.6$ \\
& b & $56.40 \pm 3.0$ & $25.87 \pm 1.6$ & $2.19 \pm 0.6$ \\
& c & $53.95 \pm 2.5$ & $13.29 \pm 1.4$ & $2.05 \pm 1.0$ \\
& d & $60.43 \pm 2.6$ & $30.67 \pm 1.5$ & $7.59 \pm 0.6$ \\
& e & $59.26 \pm 1.2$ & $26.34 \pm 1.6$ & $3.68 \pm 0.8$ \\
f & $55.86 \pm 3.0$ & $15.85 \pm 1.3$ & $2.86 \pm 0.6$
\end{tabular}

$a, b, c$ The reaction time for the substrate $d, g, h$ were $19 h$, for other substrates the reaction time were $24 \mathrm{~h}$. Enzymatic reaction conditions: molar ratio of substrate to capric acid of 1.5:1. Novozym 435 loading of $15 \mathrm{wt} \%$. Solvent-free reactions at $60^{\circ} \mathrm{C}$. Chemical reaction conditions: substrate and capric acid were added in a molar ratio of 1.5:1. Sodium methoxide loading was $15 \mathrm{wt} \%$. Methanol was used as solvent. The reaction was carried out in an ice bath for $3 \mathrm{~h}$, then the reaction was continued at room temperature for $24 \mathrm{~h}$.

$\mathrm{g}$ and $\mathrm{h}$ ), the results is different from other works (Smidt et al., 1996; Irimescu and Kato, 2004), the reason maybe from the chiral resolution need specific solvents (Rios et al., 2008; Vicente and Vicente, 2016), and control the reaction conditions (Joubioux et al., 2013).
The substrate of Table 2 could be divided into two groups. First group is the chain amino alcohols, including $\mathbf{a}, \mathbf{b}$, and $\mathbf{e}$. Second group is the aromatic amino alcohols, including $\mathbf{c}, \mathbf{d}, \mathbf{f}$, $\mathbf{g}$, and $\mathbf{h}$. For the first group, the results of the reactions were not similar to the results of other literatures. Syrén et al. found that the esters would not be formed in the lipase catalyzed acylation reactions (Syrén et al., 2012). According to our results, a small quantity of ester product was found after $24 \mathrm{~h}$ reaction. The reason might attributed to that other researches were carried out in solvents (tert-butanol), and in quite short reaction time (10 min), which was not long enough for the accumulation of ester products. For the second group, it was found that CALB illustrated better $\mathrm{N}$-acyl selectivity to the aromatic amino alcohols, and higher reaction conversion rates than chain amino alcohols. This phenomenon might be from two reasons. The products of $\mathrm{N}$-acylation would form the $p-\pi$ conjugate from benzene ring and adjacent amide group, which would increase the stability of product. Meanwhile, from the docking simulation result, it could be found that the benzene ring of substrates would bond at the active site of CALB through hydrophobic interaction, which is benefit for the enzymatic reaction. This also indicated that the structure of lipase is important for the $\mathrm{N}$-acylation selectivity.

The chemical catalysis process with sodium methoxide as catalyst was compared to lipase catalysis. The results of the chemical catalysis process (Table 2) indicated that under the reaction conditions, amide is the main product with all kinds of substrate. This is because of the amide group is more reactive than the hydroxyl group. However, since the chemical catalysis is a homogeneous reaction, the catalyst lacks regio-selectivity, the yield of ester products in chemical catalysis were much higher than that in enzymatic catalysis (Karin et al., 2013; Shirini and Khaligh, 2013; Davis and Phipps, 2017). Moreover, too much side reactions in the chemical catalysis reduced the yield of the 
amide product to significantly lower values than obtained with enzymatic catalysis.

\section{CONCLUSIONS}

In this study, the enzymatic synthesis of aromatic alkanolamides in a solvent free system was investigated. The results indicated that lipase Novozym 435 (lipase CALB) is an efficient catalyst for the reaction. The results of molecular simulation in silico illustrated that the specific structure of substrate binding site of lipase CALB determined the regio-selectivity of the lipase. Meanwhile, the results also indicated that the mechanism of specific $\mathrm{N}$-acyl selectivity of lipase was not only from intramolecular migration and proton shuttle mechanism, but also from the special structure of enzyme active site. The optimum conditions for the phenylglycolamide synthesis were determined at a molar ratio of phenylglycinol to capric acid of 1.5:1, an enzyme loading of $15 \mathrm{wt} \%$, a reaction temperature of $60^{\circ} \mathrm{C}$, with an agitation speed of $600 \mathrm{rpm}$ for $19 \mathrm{~h}$. Under these optimal reaction conditions, the yield of amide product was $89.41 \%$. The results of evaluation of different amino alcohols as substrate of the enzymatic method proved that the method has an excellent regioselectivity. Compared to the chemical method, the enzymatic synthesis exhibited high regio-selectivity, and conversions. This is a promising alternative strategy for the synthesis of aromatic alkanolamides.

\section{DATA AVAILABILITY STATEMENT}

All datasets generated for this study are included in the article/Supplementary Material.

\section{REFERENCES}

Ali, M. A., Siddiki, S. M. A. H., Kon, K., and Shimizu, K. (2013). Fe $\mathrm{F}^{3+}$-exchanged clay catalyzed transamidation of amides with amines under solvent-free condition. RSC Adv. 5:8044180449. doi: 10.1016/j.tetlet.2013.12.111

Andrea, Á., Miguel, A. E., and Susana, I. (2019). Ruthenium-catalyzed oxidative amidation of alkynes to amides. Org. Lett. 21:53465350. doi: 10.1021/acs.orglett.9b01993

Baum, I., Elsässer, B., Schwab, L. W., Loos, K., and Fels, G. (2011). Atomistic model for the polyamide formation from $\beta$-lactam catalyzed by Candida antarctica lipase B. ACS Catal. 1, 323-336. doi: 10.1021/cs1000398

Bornscheuer, U. T. (2005). Biocatalysts and enzyme technology. Focus Catal. 6, 8-8. doi: 10.1016/S1351-4180(05)71043-6

Cao, H., Deng, L., Lei, M., Wang, F., and Tan, T. (2014). The role of temperature and solvent microenvironment on the activity of Yarrowia lipolytica Lipase 2: insights from molecular dynamics simulation. J. Mol. Catal. B Enzym.109, 101-108. doi: 10.1016/j.molcatb.2014.08.013

Davis, H., and Phipps, R. J. (2017). Harnessing non-covalent interactions to exert control over regio-selectivity and site-selectivity in catalytic reactions. Chem. Sci. 8, 864-877. doi: 10.1039/C6SC04157D

Deng, L., Nie, K. L., Wang, F., and Tan, T. W. (2005). Studies on production of biodiesel by esterification of fatty acids by a lipase preparation from Candida sp. 99-125. Chinese J. Chem. Eng. 13, 529-534.

Fernandez-Perez, M., and Otero, C. (2001). Enzymatic synthesis of amide surfactants from ethanolamine. Enzyme Microb. Technol. 28, 527-536. doi: 10.1016/S0141-0229(01)00293-9

\section{AUTHOR CONTRIBUTIONS}

$\mathrm{KN}$ instructed the experiments, and helped to draft, revise the paper, and helped to revise the manuscript. LD designed and revised the experiments. MS performed the experiments and wrote the paper. FW contributed to manuscript revision, read, and approved the submitted version.

\section{FUNDING}

This work was supported by the National Key Research and Development Program of China (2016YFD0400601, 2017YFB0306800, 2017YFD0400603, 2017YFB0306801, 2017YFB0306904), National Natural Science Foundation of China (21978017), the Amoy Industrial Biotechnology R\&D and Pilot Conversion Platform (3502Z20121009).

\section{ACKNOWLEDGMENTS}

The authors wish to express thanks for the supports from the National Key Research and Development Program of China (2016YFD400601, 2017YFB0306800, 2017YFD0400603, 2017YFB0306801, 2017YFB0306904), National Natural Science Foundation of China (21978017), the Amoy Industrial Biotechnology R\&D and Pilot Conversion Platform (3502Z20121009).

\section{SUPPLEMENTARY MATERIAL}

The Supplementary Material for this article can be found online at: https://www.frontiersin.org/articles/10.3389/fbioe.2019. 00486/full\#supplementary-material

Fernandez-Perez, M., and Otero, C. (2003). Selective enzymatic synthesis of amide surfactants from diethanolamine. Enzyme Microb. Technol. 33, 650-660. doi: 10.1016/S0141-0229(03)00184-4

Fodor, G., and Kiss, J. (1950). Acyl migration O-N in the diastereomeric 2-aminocyclohexyl benzoates. J. Am. Chem. Soc. 72, 3495-3497. doi: $10.1021 / \mathrm{ja} 01164 \mathrm{a} 049$

Garcia-Alles, L. F., and Gotor, V. V. (2015). Alcohol inhibition and specificity studies of lipase B from candida antarctica in organic solvents. Biotechnol. Bioeng. 59, 163-170. doi: 10.1002/(sici)1097-0290(19980720)59:2<163::aid-bit4>3.0.co;2-f

Gorman, L. A., and Dordick, J. S. (2010). Organic solvents strip water off enzymes. Biotechnol. Bioeng. 39, 392-397. doi: 10.1002/bit.260390405

He, X. X., and Schuchman, E. H. (2018). Ceramide and ischemia/reperfusion injury. J. Lipids Jan. 21:3646725. doi: 10.1155/2018/3646725

Irimescu, R., and Kato, K. (2004). Lipase-catalyzed enantioselective reaction of amines with carboxylic acids under reduced pressure in non-solvent system and in ionic liquids. Tetrahedron Lett. 45, 523-525. doi: 10.1016/j.tetlet.2003.10.210

Jiang, F. Z., Jin, K., Huang, S. Y., Bao, Q., Shao, Z., R., Hu, X, Q., et al. (2016). Liposomal C6 ceramide activates protein phosphatase 1 to inhibit melanoma cells. PLoS ONE 11:e0159849. doi: 10.1371/journal.pone.0159849

Joubioux, F. L., Bridiau, N., Henda, Y. B., Achour, O., Graber, M., and Maugar, T. (2013). The control of Novozym $(\mathbb{R} 435$ chemoselectivity and specificity by the solvents in acylation reactions of amino-alcohols. J. Mol. Catal. B-Enzym. 95, 99-110. doi: 10.1016/j.molcatb.2013.06.002

Karin, E. M., Johnston, E. V., Oscar, V., Gustafson, K. P. J., Mozaffar, S., and Cheuk-Wai, T. (2013). Co-immobilization of an enzyme and a metal into 
the compartments of mesoporous silica for cooperative tandem catalysis: an artificial metalloenzyme. Angew. Chem. Int. Ed. Engl. 125, 14256-14260. doi: 10.1002/ange.201306487

Kirchner, G., Scollar, M. P., and Klibanov, A. M. (1985). Resolution of racemic mixtures via lipase catalysis in organic solvents. Jam. Chem. Soc. 107, 7072-7076. doi: 10.1021/ja00310a052

Lee, C. H., and Parkin, K. L. (2001). Effect of water activity and immobilization on fatty acid selectivity for esterification reactions mediated by lipases. Biotechnol. Bioeng. 75:219. doi: 10.1002/bit.10009

Levinson, W. E., Kuo, T. M., and Kurtzman, C. P. (2005). Lipase-catalyzed production of novel hydroxylated fatty amides in organic solvent. Enzyme Microb. Tech. 37, 126-130. doi: 10.1016/j.enzmictec.2005.02.001

Liñares, G. G., Mañez, P. A., and Baldessari, A. (2014). Lipase-catalyzed synthesis of substituted phenylacetamides: hammett analysis and computational study of the enzymatic aminolysis. Eur. J. Org. Chem. 29, 6439-6450. doi: 10.1002/ejoc.201402749

Long, Z., Hellgren, L. I., and Xuebing, X. J. (2006). Enzymatic production of ceramide from sphingomyelin. J. Biotechnol. 123, 93-105. doi: $10.1016 /$ j.jbiotec. 2005.10 .020

Martinek, K., and Semenov, A. N. (2002). Enzyme catalysis in organic synthesis. Focus Catal. 8, 6-8. doi: 10.1016/S1351-4180(02)01161-3

Martínková, L., Vejvoda, V., and Kren, V. (2008). Selection and screening for enzymes of nitrile metabolism. J. Biotechnol. 133, 318-326. doi: 10.1016/j.jbiotec.2007.10.011

Matsui, T., Kondo, T., Nishita, Y., Itadani, S., Tsuruta, H., Fujita, S., et al. (2002). Highly potent inhibitors of tnf- production. Part II: metabolic stabilization of a newly found chemical lead and conformational analysis of an active diastereoisomer. Bioorg. Med. Chem. 10, 3787-3805. doi: 10.1016/S0968-0896(02)00380-2

Maugard, T., Remaud-Simeon, M., Petre, D., and Monsan, P. (1997). Lipase-catalyzed chemoselective n-acylation of amino-sugar derivatives in hydrophobic solvent: acid-amine ion-pair effects. Tetrahedron 28, 7587-7594. doi: 10.1016/S0040-4020(97)00450-X

Morad, S. A. F., and Cabot, M. C. (2013). Ceramide-orchestrated signalling in cancer cells. Nat. Rev. Cancer 13, 51-65. doi: 10.1038/nrc3398

Müller, H., Hellgren, L. I., Olsen, E., and Skrede, A. (2004). Lipids rich in phosphatidylethanolamine from natural gas-utilizing bacteria reduce plasma cholesterol and classes of phospholipids: a comparison with soybean oil. Lipids 39, 833-841. doi: 10.1007/s11745-004-1304-5

Plastina, P., Meijerink, J., Vincken, J. P., Gruppen, H., Witkamp, R., and Gabriele, B. (2009). Selective synthesis of unsaturated n-acylethanolamines by lipasecatalyzed n-acylation of ethanolamine with unsaturated fatty acids. Lett. Org. Chem. 6, 444-447. doi: 10.2174/157017809789124885

Prasad, A. K., Husain, M., Singh, B. K., Gupta, R. K., Manchanda, V. K., Olsen, C. E., et al. (2005). Solvent-free biocatalytic amidation of carboxylic acids. Tetrahedron Lett. 46, 4511-4514. doi: 10.1016/j.tetlet.2005.04.121

Ragupathy, L., Ziener, U., Dyllickbrenzinger, R., Von Vacano, B., and Landfester, K. (2012). Enzyme-catalyzed polymerizations at higher temperatures: synthetic methods to produce polyamides and new poly(amide-co-ester)s. J. Mol. Cata B-Enzym 76, 94-105. doi: 10.1016/j.molcatb.2011.11.019

Rios, A. P. D. L., Hernández-Fernández, F. J., Tomás-Alonso, F., Gómez, D., and Víllora, G. (2008). Synthesis of flavour esters using free candida antarctica lipase b in ionic liquids. Flavour Frag J. 23, 319-322. doi: 10.1002/ ffj. 1884

Schwab, L. W., Kloosterman, W. M., Konieczny, J., and Loos, K. (2013). Papain catalyzed synthesis of protected amino acid amides. J. Renew. Mater. 1, 73-78. doi: 10.7569/JRM.2012.634102

Shirini, F., and Khaligh, N. G. (2013). A succinimide- N -sulfonic acid catalyst for acetylation reactions in absence of a solvent. Chinese J. Catal. 34, 695-703. doi: 10.1016/S1872-2067(11)60499-3

Skorey, K., Somayaji, V., Brown, R., and Ball, R. (1987). Molecular structure of 3, 4, 5-trihydro-2-oxo-1, 5-ethanobenzazepine and its reaction with. Beta.-amino alcohols as a model for the acylation step of the serine proteases. J. Org. Chem. 51, 4866-4872. doi: 10.1021/jo00375a020

Slotema, W. F., Georgina, S., David, G., Straathof, A. J. J., and Alain, M. (2003). Economically pertinent continuous amide formation by direct lipase-catalyzed amidation with ammonia. Biotechnol. Bioeng. 82, 664-669. doi: 10.1002/bit.10613

Smidt, H., Fischer, A., Fischer, P., and Schmid, R. D. (1996). Preparation of optically pure chiral amines by lipase-catalyzed enantioselective hydrolysis of N-acyl-amines. Biotechno Tech. 10, 335-338. doi: 10.1007/BF00173249

Stavila, E., Alberda van Ekenstein, G. O. R., and Loos, K. (2013). Enzyme-catalyzed synthesis of aliphatic-aromatic oligoamides. Biomacromolecules 14, 1600-1606. doi: 10.1021/bm400243a

Sugiyama, S., Honda, M., Higuchi, R., and Komori, T. (2010). ChemInform abstract: biologically active glycosides from asteroidea. Part 26. stereochemistry of the four diastereomers of ceramide and ceramide lactoside. J. Org. Chem. 22, 349-356. doi: 10.1002/chin.199132253

Syrén, P. O., Joubioux, F. L., Henda, Y. B., Maugard, T., Hult, K., and Graber, M. (2012). Proton shuttle mechanism in the transition state of lipase-catalyzed N-acylation of amino alcohols. ChemCatChem 5, 1842-1853. doi: $10.1002 /$ cctc. 201200751

Tan, T. W. (2010). Biodiesel production with immobilized lipase: a review. Biotechnol. Adv. 28, 628-634. doi: 10.1016/j.biotechadv.2010.05.012

Tan, T. W., Zhang, M., Wang, B. W., Ying, C. H., and Deng, L. (2003). Screening of high lipase producing Candida sp. and production of lipase by fermentation. Process Biochem. 29. 459-465. doi: 10.1016/S0032-9592(03)00091-8

Toshiaki, M., Takashi, K., Yoshitaka, N., Satoshi, I., Shingo, N., Nagashige, O., et al. (2002). Highly potent inhibitors of TNF-alpha production. Part I: discovery of new chemical leads and their structure-activity relationships. Bioorg. Med. Chem. 10, 3757-3786. doi: 10.1016/S0968-0896(02)00381-4

Tufvesson, P., Annerling, A., Hatti-Kaul, R., and Adlercreutz, D. (2006). Solventfree enzymatic synthesis of fatty alkanolamides. Biotechnol. Bioeng. 97, 447-453. doi: 10.1002/bit.21258

Vicente, G. (2002). Lipases and (R)-oxynitrilases: useful tools in organic synthesis. J. Biotechnol. 96, 35-42. doi: 10.1016/S0168-1656(02)00035-4

Vicente, G. F., and Vicente, G. (2016). "Enantioselective acylation of alcohol and amine reactions in organic synthesis," in Green Biocatalysis, ed R. N. Patel (Oviedo: John Wiley and Sons Inc.), 231-266. doi: 10.1002/9781118828083.ch9

Wang, X., Yang, C., Yun, M., Jin, Q., and Wang, X. J. (2015). Lipozyme 435catalyzed synthesis of eicosapentaenoyl ethanolamide in a solvent-free system. Mol. Catal. 122, 233-239. doi: 10.1016/j.molcatb.2015.09.016

Wang, X. S., Wang, X. G., and Wang, T. (2012). Synthesis of oleoylethanolamide using lipase. J. Agric. Food Chem. 60, 451-457. doi: 10.1021/jf203629w

Wang, X. S., Wang, X. G., and Wang, T. (2013). Enrichment of arachidonic acid for the enzymatic synthesis of arachidonoyl ethanolamide. J. Am. Oil Chem Soc. 90, 1031-1039. doi: 10.1007/s11746-013-2247-7

Xiao, P., Zhang, S., Ma, H., Zhang, A., Lv, X., and Zheng, L. (2013). Stereoselective synthesis of caffeic acid amides via enzyme-catalyzed asymmetric aminolysis reaction. J. Biotechnol. 168, 552-559. doi: 10.1016/j.jbiotec.2013.09.004

Zaks, A., and Klibanov, A. M. (1984). Enzymatic catalysis in organic media at $100^{\circ}$ C. Science 224, 1249-1251. doi: $10.1126 /$ science. 6729453

Conflict of Interest: The authors declare that the research was conducted in the absence of any commercial or financial relationships that could be construed as a potential conflict of interest.

Copyright (c) 2020 Sun, Nie, Wang and Deng. This is an open-access article distributed under the terms of the Creative Commons Attribution License (CC BY). The use, distribution or reproduction in other forums is permitted, provided the original author(s) and the copyright owner(s) are credited and that the original publication in this journal is cited, in accordance with accepted academic practice. No use, distribution or reproduction is permitted which does not comply with these terms. 